\title{
Hipertensão Arterial Sistêmica. Uma Doença Programada no Útero?
}

\author{
Sandra S. Mattos
}

Recife, PE

\begin{abstract}
A hipertensão arterial sistêmica (HAS) é fator de risco para a doença coronária e o acidente vascular cerebral.

Desde os estudos de Framingham ${ }^{1}$, há quase meio século, cardiologistas e clínicos gerais travam uma batalha preventiva contra a HAS e a doença aterosclerótica coronária (DAC).
\end{abstract}

Mudanças de hábitos higieno-dietéticos, controle dos níveis de pressão arterial (PA), implementação de programas de condicionamento físico, redução nas jornadas de trabalho com melhor aproveitamento de momentos de lazer são os principais aspectos preventivos enfatizados.

Ainda assim, a morbi-mortalidade decorrente da HAS e da DAC representam o maior problema de Saúde Pública em todo o mundo.

Desde a década de 50, estudos anatomopatológicos em jovens soldados mortos nas guerras da Coréia e Vietnã alertaram para o desenvolvimento precoce da doença. Obstrução coronária $>50 \%$ do lúmen dos vasos foi observada em $15 \%$ desses jovens com idade média de 22 anos na Coréia ${ }^{2}$. Doença obstrutiva em dois ou três vasos foi encontrada em $26 \%$ e $4 \%$, respectivamente, nos casos estudados no Vietnã ${ }^{3}$.

Alterações ateromatosas na aorta foram documentadas em crianças com 10 anos de idade ${ }^{4}$.

A observação de que mais e mais adultos jovens desenvolviam HAS e coronariopatias levou Paul Dudley White ${ }^{5,6}$, nos anos 70, a propor uma "Cruzada da Criança: para modificar o estilo de vida das crianças do nosso século".

Até hoje, ainda são poucos os centros de Cardiologia Pediátrica que desenvolvem trabalhos em medicina preventiva.

Campanhas de saúde pública, envolvendo os órgãos de telecomunicação, raramente abordam a importância da educação preventiva para esta epidemia.

Observações mais recentes ampliaram o nosso conhe-

Instituto do Coração de Pernambuco - Real Hospital Português - Recife Correspondência: Sandra S. Mattos - Unidade de Cardiologia Materno-Fetal Instituto do Coração de PE - Av. Agamenon Magalhães, S/N - 52090-900 - Recife, PE

Recebido para publicação em 28/5/97

Aceito 25/6/97 cimento sobre os mecanismos de base para o desenvolvimento da HAS na vida adulta.

Em 1990, Barker e $\mathrm{col}^{7}$, observaram que PA na vida adulta correlaciona-se inversamente com o peso ao nascimento. Esta correlação á ainda mais evidente, quando a relação fetal/peso placentário está alterada em favor da placenta (Barker e col, 1991) ${ }^{8}$. Os autores sugeriram que a relação a longo prazo entre crescimento fetal alterado e PA subseqüente, e a sua importância nas diferenças qualitativas no crescimento, sugere que a fisiopatologia da HAS pode estar baseada em crescimento tecidual comprometido por um ambiente adverso durante um período crítico, possivelmente curto, do desenvolvimento fetal.

Konje e col, em 1996 ${ }^{9}$, estudando os diâmetros renais em fetos normais e naqueles pequenos para a idade gestacional (PIG) observaram menores diâmetros dos rins nos fetos PIG. Nestes, ainda, os níveis de renina plasmática documentada na veia umbilical, ao nascimento, estavam significantemente mais elevados do que nos neonatos com desenvolvimento normal.

Estas observações apontam para uma possível "programação intra-uterina" da PA, e ampliam sobremaneira as áreas de atuação da cardiologia fetal.

A cardiologia fetal vem sendo praticada há pouco mais de uma década. Suas áreas de atuação incluem a detecção das cardiopatias congênitas estruturais, e alterações hemodinâmicas, funcionais e do ritmo cardíaca fetal. $\mathrm{O}$ manuseio destas anomalias pode iniciar-se ainda intra-útero, como nas reversões das arritmias cardíacas, ou ser planejado para após o nascimento. O acompanhamento psicológico e o aconselhamento genético são alguns dos principais aspectos do trabalho interdisciplinar, fundamental para a prática da especialidade.

O trabalho interdisciplinar com obstetras, fetologistas, ultra-sonografistas, nutricionistas e geneticistas na detecção precoce de alterações na circulação materno-feto-placentária, suas influências na organogênese e possíveis correlações com o desenvolvimento da HAS e da DAC constituise uma nova e excitante área de atuação da cardiologia fetal.

Seria este, um dos ramos da cardiologia preventiva do $3^{\circ}$ milênio? 


\section{Referências}

1. Kannel WB, Gordon T - The Framingham Study: an epidemiological investigation of cardiovascular disease, section 30 . Some characteristics related to the incidence of cardiovascular disease and death: The Framingham Study. 18-year follow-up. Washington DC, Government Printing Office, 1974.

2. Enos WF, Beyer JC, Holmes RH - Pathogenesis of coronary disease in American soldiers kiled in Korea. JAMA 1955; 158: 912-6.

3. McNamara JJ, Molot MA, Stremple JF, Cutting RT - Coronary artery disease in combat casualities in Viet Nam. JAMA 1971; 216: 1185-7.

4. Strong JP, MCGil HC Jr - The pediatric aspect of atherosclerosis. J Atheroscler Res 1969; 9: 251-65.

5. White PD - The tardy growth of preventive cardiology. Am J Cardiol 1972; 29: 886-8.
6. White PD - The early infancy of preventive cardiology. Trans Am Clin Climatol Assoc 1973; 84: 17-21.

7. Barker D, Bull A, Osmond C, Simmonds S-Fetal and placental size and the risk of hypertension in adult life. Br Med J 1990; 301: 259-62.

8. Barker D, Godfrey K, Osmond C, Bull A - The relation of fetal length, ponderal index and head circunference to blood pressure and the risk of hypertension in adult life. Paediatrics and Perinatal Epidemiology 1991; 6: 35-44.

9. Konje JC, Bell SC, Morton JJ, de Chazal R, Taylor DJ - Human fetal kidney morphometry during gestational and the relationship between weight, kidney morphometry and plasma active renin concentration ar birth. Clin Sci Colch 1996; 91: 169-75. 